wise appears to be very similar to it. Yet NGC 604 is located a considerable distance from the nucleus of M33.

Mathewson: It may be possible for some galaxies to have two nuclei.

Davies: The density of sources in your $1410 \mathrm{Mc} / \mathrm{s}$ map appeared to be as large outside the Clouds as inside. You associate the radio sources inside the Clouds with optical objects. What is the origin of the sources outside the periphery of the Clouds?

Mathewson: The density of intense sources is higher inside the main body of the Clouds than outside. At the moment I have not attempted to associate the optically unidentified sources with the Clouds, although the sources with thermal-type spectral indices and the extended nonthermal sources found in the outlying regions may well be profitably investigated with optical telescopes.

Ambartsumian: What is the ratio of integrated intensities of nonthermal emission of the central bar and of the region surrounding 30 Doradus, say at $408 \mathrm{Mc} / \mathrm{s}$ ?

Mathewson: The integrated emission at $408 \mathrm{Mc} / \mathrm{s}$ from the central nonthermal bar is about $150 \times 10^{-26} \mathrm{Wm}^{-2}(\mathrm{c} / \mathrm{s})^{-1}$ and from the 30 Doradus region about $100 \times 10^{-26} \mathrm{Wm}^{-2}(\mathrm{c} / \mathrm{s})^{-1}$.

Arp : Can you say whether the general polarization activity in the region of the Magellanic Clouds is higher than in corresponding galactic latitudes in much different directions?

Mathewson: Healey, Milne, and myself have commenced a general "background" polarization survey at $408 \mathrm{Mc} / \mathrm{s}$ of regions south of declination $30^{\circ}$. However, at the moment not a wide enough area in latitude has been surveyed to answer your question.

Oort: This is a tremendous amount of material of very great interest which you have presented. In reply to Dr. Arp's question, concerning the foreground polarization you observe in the general direction of the Small Cloud, I want to point out that distribution of the galactic polarization in high latitudes is so chaotic that one cannot hope to predict what it would be in front of the Small Cloud.

Have you any information on the direction of the magnetic field in the bar of the Large Cloud?

Mathewson: The observed position angle of the plane of polarization has not yet been corrected for Faraday rotation in the ionosphere. If the direction of the magnetic field obtained from the radio measurements agreed with that derived from the optical polarization measurements, one would have more confidence in associating the polarized radio emission with the Clouds.

Buscombe: Has any comparison been made of the space distribution of radio emission in the SMC with $(a)$ early-type supergiants, and $(b)$ the star clusters, which delineate quite a different distribution both in position and velocity from the bright stars?

Mathewson: No such comparison has been made.

\title{
55. NOTES ON THE STRUCTURE OF THE SMC AS OBSERVED IN 21-CM LINE RADIATION FROM NEUTRAL HYDROGEN
}

\author{
J. V. Hindman \\ CSIRO Radiophysics Laboratory, Sydney
}

\section{Introduction}

Earlier surveys of the line radiation from neutral hydrogen in the Magellanic Clouds (Kerr, Hindman, and Robinson 1954; Hindman, Kerr, and McGee 1963; Hindman et al. 1963) have shown that the amount of gas associated with these extragalactic bodies is relatively large. The gas appears widespread, surrounding the stellar bodies in a single continuous and very tenuous envelope with a marked bridge in the vicinity of the optical wing of the SMC linking the two main concentrations. 
The Small Cloud profiles have also been observed to have double peaks, over a considerable area, which were separated by a roughly constant velocity difference of approximately $30 \mathrm{~km} / \mathrm{sec}$, and it was suggested that these peaks may originate in two substantially separate masses of gas.

Up to this stage the investigation of the Cloud system was performed with small aerials having beamwidths of 1.5 and 2.2 , thus giving a smoothed picture of the two objects which were only of the order of 5 to 10 beamwidths in diameter.

A new survey of the central region of the SMC has been undertaken with the Parkes 210-foot aerial with a beamwidth of only a quarter of a degree. Much more detail has been observable than in the previous surveys, both in sky distribution and consequently in velocity, since the velocity data have been averaged over a smaller volume of gas.

The observational work is substantially complete except for essential checking of reference areas and observations of special features. Only a small amount of the data (some 1500 of a total of nearly 4000 profiles) has been reduced and this work is continuing.

The present paper reports on some of the features apparent in the reductions so far completed.

\section{Observations}

The observations were made using the 48-channel hydrogen-line receiver of the Radiophysics Division of CSIRO, described by McGee and Murray (1963), in conjunction with the Parkes 210-foot telescope. This combination gives the following observing parameters:

Beamwidth: 15 min of arc

Receiver channel bandwidth: $37 \mathrm{kc} / \mathrm{s} \quad 8.0 \mathrm{~km} / \mathrm{sec}$

Receiver channel spacing: $33 \cdot 2 \mathrm{kc} / \mathrm{s} \quad 7.0 \mathrm{~km} / \mathrm{sec}$

Receiver velocity range: $\pm 170 \mathrm{~km} / \mathrm{sec}$ centred on $220 \mathrm{~km} / \mathrm{sec}$.

The method of observing was to track the aerial across the sky, usually on a constant declination at a rate of 1 degree in $20 \mathrm{~min}$ of time or $\frac{1}{10}$ th degree in $2 \mathrm{~min}$ of time; the receiver records one profile every $2 \mathrm{~min}$ and thus we had slightly more than two profiles per beamwidth.

The zero for the profiles reduced so far is taken from observations at R.A. $00^{\mathrm{h}} 00^{\mathrm{m}}$ and the same declination as the run being reduced. Any error introduced in this way is quite small and will finally be eliminated by measurements relative to the South Celestial Pole.

\section{Results}

(a) Integrated Brightness.-Figure 1 shows contours of integrated brightness over the central region of the SMC and there is clearly good general agreement between the features of this map and the optical distribution. The main bar with the concentration at the southern end is clearly seen, as is the wing region extending toward the LMC, and there is rough agreement between concentrations of $\mathrm{HI}$ and the positions of HII emission regions. 
One such region, centred on dec. $-73^{\circ} 20^{\prime}$ and R.A. $00^{\mathrm{h}} 48^{\mathrm{m}}$, shows a very intense peak in the profile which rises to $T_{A} 130^{\circ} \mathrm{K}$, whereas in the other areas where the profile intensity shows a peak the temperature is around $T_{A} 90^{\circ} \mathrm{K}$. This very high temperature peak is associated with a concentration of HII emission regions which coincide with peaks in the 1410 continuum (Mathewson and Healey, this volume, paper 53). The HI peak actually lies between two continuum centres, suggesting that we are seeing a deficiency of $\mathrm{HI}$ in the regions of strong ionization. The high temperature of this peak, which is very little less than the highest temperature

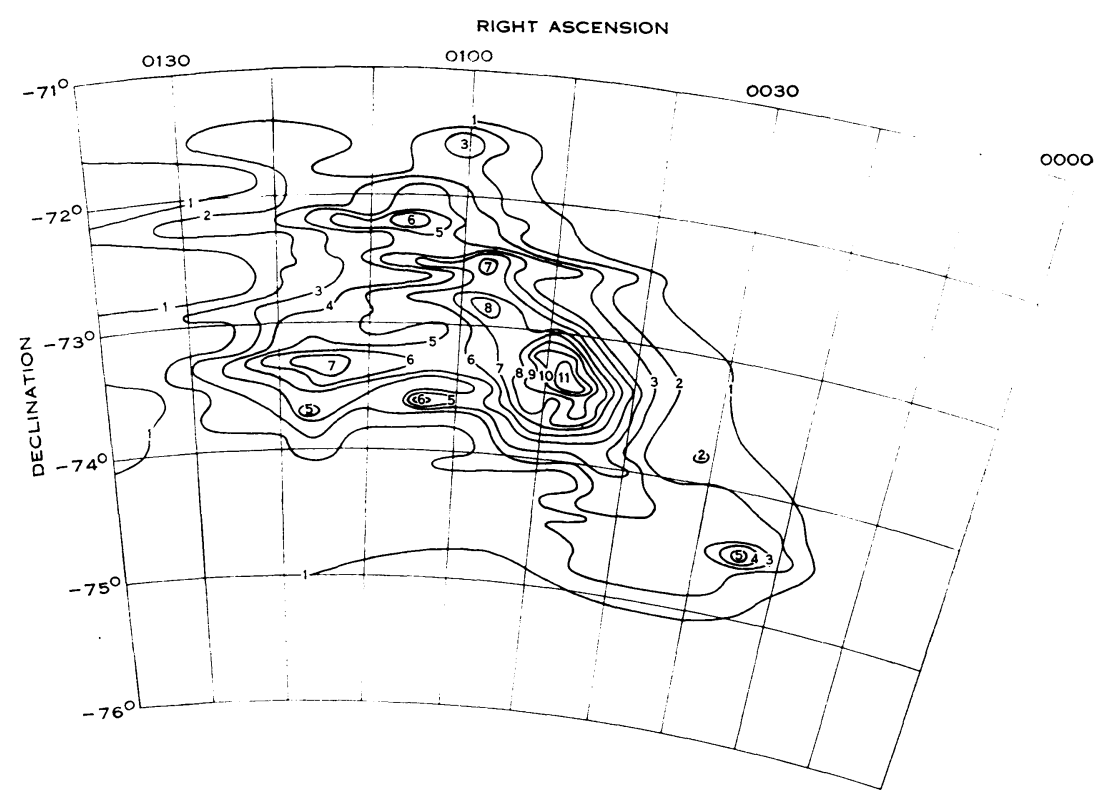

Fig. 1.-Contours of integrated brightness of $\mathrm{H}$-line radiation in the region of the SMC. Contour interval $=1 \cdot 7 \times 10^{-15} \mathrm{Wm}^{-2}$ steradian $^{-1}$.

regions of the Galaxy, suggests the possibility of increased kinetic temperature of the gas in regions of high intensity showing a large concentration of hot stars and emission regions.

(b) Profiles.-The most striking feature of the profiles is that in $30 \%$ of them, there are two distinct and widely separated peaks. Closer examination shows that in fact there are a number of fairly easily recognized peaks which can be traced readily from profile to profile, each varying in intensity more or less independently across the area of the Small Cloud system. When the intensities of the individual peaks are plotted the result is the distribution shown in Figure 2. It is readily seen that the area on the sky occupied by individual peaks of the profile is quite different and if one examines the shapes of the various areas it is seen that the main body or bar of the optical distribution agrees fairly well with area $\mathrm{A}$ whilst half of area $\mathrm{C}$ fits the position of the wing very well. Area B likewise corresponds with the intense blue region of the lower end of the optical bar. Two salient facts are noted in an examination of the individual velocity peaks: (i) They are in general quite widely 
separated in velocity; (ii) there appears to be no suggestion in any of the profiles of the peaks at various velocities tending to shift systematically so as to merge with each other.

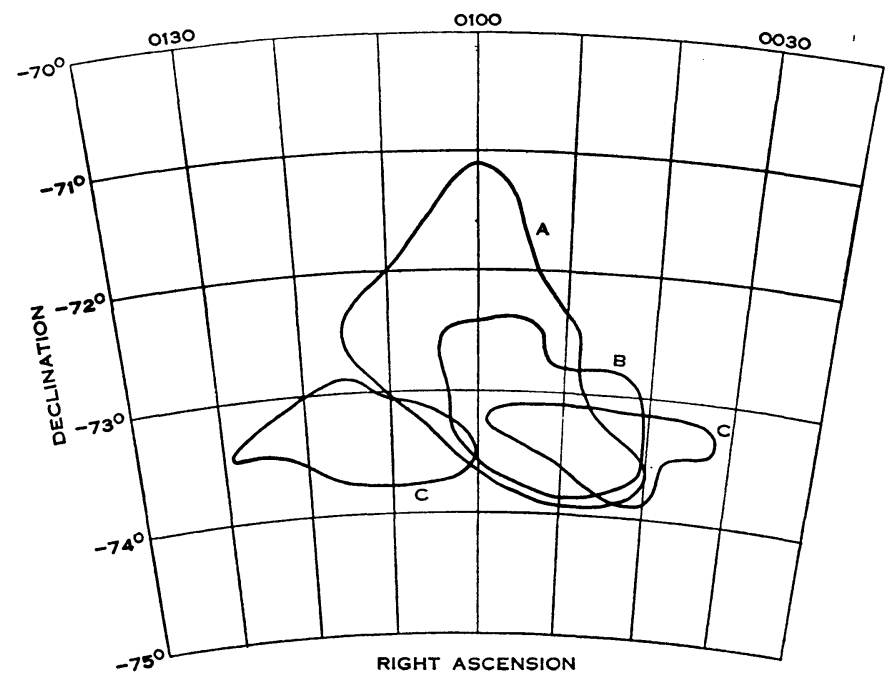

Fig. 2.-Outlines of areas in which discrete profile peaks are found. The outline is in each case at the point where the peak intensity of a given peak is $20^{\circ} \mathrm{K}\left(T_{A}\right)$.

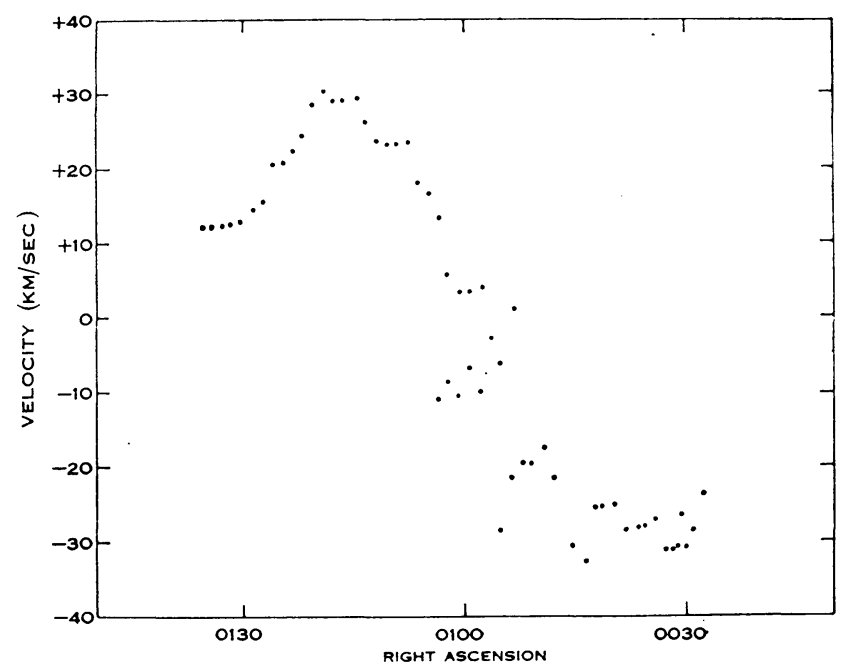

Fig. 3.-Rotation curve for the gas in the SMC which is distributed in the region of the wing of the cloud and overlapping in the main bar toward the southern end.

These facts, coupled with the widely differing distribution in the plane of the sky, lead to the suggestion that the regions concerned are in fact independent bodies of gas. 
Examination of the variations of velocity across the regions delineated above may be of help in deciding what the motions of the various sections are. One set of data has been roughly examined, that for dec. $-73^{\circ} 24^{\prime}$, which lies almost along the axis of the elongated region C. Figure 3 shows the resulting velocity curve, which is very suggestive of differential rotation. The low velocity end of the curve is less well defined than the high velocity end, but this is the region where the various velocity regions overlap and the true peak velocity may be displaced owing to lack of frequency resolution.

The detailed velocity data for other peaks have not been examined yet; all that can be said is that for region $A$ the velocity range is of the order of $20 \mathrm{~km} / \mathrm{sec}$ and for region $B$ it is very narrow, perhaps only $10 \mathrm{~km} / \mathrm{sec}$.

\section{Mass of Hydrogen}

A calculation of the total hydrogen mass for the central region of the SMC has been made. The figure obtained, $2 \times 10^{8} M \odot$, is somewhat less than that given for the SMC nucleus from the broad beam surveys. This is because the previous figures included a very considerable contribution from the link between the Clouds extending out to $03^{\mathrm{h}} 00^{\mathrm{m}}$ R.A.

The mass of neutral hydrogen has been estimated for the elongated system $\mathrm{C}$ of Figure 2 and this comes to $0.8 \times 10^{8} M \odot$.

\section{Conclusion}

In conclusion it is of interest to list the main features so far dealt with in this investigation and to note some features of the observational data which have not yet been discussed.

1. There is a single very high temperature peak in the profiles near R.A. $00^{\mathrm{h}} 48^{\mathrm{m}}$, dec. $-73^{\circ} 20^{\prime}$, which is almost $50 \%$ higher than any of the other peak temperatures recorded.

2. The profiles show systematic differences in velocity between clearly defined peaks over extensive areas of the SMC region.

3. Some very sharp profile features have been noted with halfwidths probably less than $7 \mathrm{~km} / \mathrm{sec}$.

4. The surrounding envelope region shows a very patchy distribution both in intensity and frequency of the profiles, suggesting an area of small discrete clouds.

5. Correspondence with optical features is varied and some comparison point other than positional superposition is urgently needed. This could possibly be best provided at present by optical nebula velocities for the various emission regions.

\section{References}

Hindmax, J. V., Kerr, F. J., and McGee, R. X. (1963).-Aust. J. Phys. 16: 570-83.

Hindman, J. V., McGee, R. X., Carter, A. W. L., Holmes, E. C. J., and Beard, M. (1963).Aust. J. Phys. 16 : 552-69.

Kerr, F. J., Hindiax, J. V., and Robissox, B. J. (1954).-Aust. J. Phys. 7 : 297-314.

McGee, R. X., and Murray, J. D. (1963).-Proc. I.R.E. (Aust.) 24 : 191-6. 


\section{Discussion}

Lindblad: May I ask if you have tried to make an estimate of the total mass from the rotation curve?

Hindman: The results reported have been reduced only in the last two months and $I$ have not yet attempted a solution for total mass.

Arp : Is there any reason to say that the velocity difference observed cannot be explained by a rotational model? That is, it is not necessary to conclude that the SMC is expanding in its different parts at this rate.

Hindman: The full $40 \mathrm{~km} / \mathrm{sec}$ may not be the difference between the systematic velocities for the two main gas masses, but there will be a substantial line-of-sight component, which is unlikely to be explained by a rotation velocity which would have to be very large since the axis of double the system must be close to the line of sight.

Christiansen: Does the evidence point toward a disk or bar in the SMC?

Hindman: I wouldn't argue at this stage with either a disk or a bar, but since the curve shows differential rotation, the final form of the object must be disk-like.

Walraven: The two wings extending from the Small Cloud are also prominent with respect to the number of stars. What puzzles me is that, while the wing towards larger right ascension contains HII regions and young $\mathrm{OB}$ stars, the other wing contains mainly more evolved stars and no nebulae.

Hindman: The region you mention is the place where the systems overlap and so the older stars could well belong to the main optical stellar distribution lying in front of the elongated mass of gas.

The elongated region also has relatively few stars in the wing region and would not be expected to contribute a very high proportion of the stars in the overlapping region.

de Vaucouleurs: The new HI total brightness contours have a striking resemblance to the star count contours at $M=16 \cdot 0$ (see $A . J .60: 221$ (1955)), including some minor details such as the narrow "tongue" south-preceding knot $\mathrm{K}_{1}$ and well seen in the gradient contours (Fig. 8 , loc. cit.). The latter region coincides with one of the double-peak areas outlined by Mr. Hindman.

Kerr: If the various entities have been moving apart in the line of sight for reasonable periods of time, the group must be very extended in depth. Have you made any estimates of this effect?

Hindman: Yes, the expansion or contraction rate in the line of sight is of the order of 3-4 kpc in $10^{8}$ years, i.e. the depth dimension could be approximately equal to the width of the system viewed on the sky. On the other hand, if $10^{9}$ years of expansion has been involved, then the separation would be of the same order as the spacing between LMC and SMC.

It is unlikely of course that the velocity will remain constant.

Oort: In view of the gravitational field as indicated by the rotational motions which you derived, one could hardly expect an expansion to continue for a very long time. It therefore seems that there is little point in speculating about the distances which would be reached if the differential radial motions as indicated by the double peaks would continue for periods of more than $10^{8}$ years.

Blaauw: With reference to the double-peaked profiles which one finds over extended areas, it may not be necessary to consider these as a large-scale expansion. They may be interpreted as the superposition of a number of smaller-scale regions with similar expansion velocities. As long as the mean velocities of the matter in these regions of local expansion do not scatter by more than, say $10 \mathrm{~km} / \mathrm{sec}$, one would still in their superposition observe the double peaks. This interpretation avoids the conclusion that, with a reasonable age of the Cloud, one arrives at an excessive dimension in the line of sight. feature.

Local regions of expansion of e.g. 200 pc diameter would not seem to be an unlikely

Hindman : This seems a very unlikely explanation to me; smoothing by the aerial beam and by the fact that presumably these regions would not be expanding or contracting only in the line 
of sight would generally give quite normal smooth profiles for such a model without any separation of peaks. Further, the region to the north of the object where only a single narrow peak appears would require a fairly sudden change-over from these expanding regions to a smooth non-expanding distribution of gas.

Thackeray: Is it quite impossible to explain the double peaks in terms of central absorption in the profile?

Hindman: Two possible types of absorption may be considered: (1) Absorption due to a continuum source which is not observed at $1410 \mathrm{Mc} / \mathrm{s}$. (2) Self-absorption due to temperature variation in the gas. This seems unlikely for two reasons: Firstly, the minimum between the peaks falls to a very low value, less than $10^{\circ} \mathrm{K} T_{A}$, and this is a very low temperature for gas in clouds having a velocity dispersion of 25 to $30 \mathrm{~km} / \mathrm{sec}$. Secondly, the half of the area to the north of the system shows only a single narrow feature; it seems unlikely that low-temperature gas should cease to exist or that the velocity range should suddenly contract to less than half that applicable to the rest of the region.

I feel that these explanations require much more imagination than to accept the separated masses of gas.

Oort: You discussed the possibility that the phenomenon of the two peaks might be due to an expansion. In view of the total mass of the Cloud it seems that an expansion with a velocity of $20 \mathrm{~km} / \mathrm{sec}$ could hardly persist for a long time. The matter would be drawn back again.

Hindman: My remarks on this point were in answer to Dr. Kerr's question and I agree entirely with Professor Oort that there is good reason to be suspicious of the reasoning involved.

Gascoigne: To clarify, I take it that your results imply that the neutral $\mathrm{H}$ in the SMC appears divided between about six large parts, the masses of each of which can in principle at least be determined from the profiles.

Hindman: Yes, there are three main regions. The mass determination is not always straightforward because of the necessity to separate the gas associated with overlapping peaks; higher-frequency resolution may assist in this.

\title{
56. OPTICAL EVIDENCE ON THE KINEMATICS OF THE MAGELLANIC CLOUDS
}

\author{
M. W. Feast \\ Radcliffe Observatory
}

Some while ago radial velocities were published for 112 stars in both Magellanic Clouds from Radcliffe Cassegrain spectrograms (Feast, Thackeray, and Wesselink 1960).

An analysis of the stellar velocities (Feast, Thackeray, and Wesselink 1961) provided interesting information on the rotation and velocity dispersion in the Clouds but more data were needed to settle various doubtful points. Shortly after the installation of the Radcliffe coudé spectrograph it was found possible to obtain spectra of the brighter emission nebulae in the Clouds at $15 \AA / \mathrm{mm}$. The sharpness of the emission lines and the relatively high dispersion yields velocities of high weight. During the years 1960-63, 48 coudé spectra of 42 regions of nebulosity in the Large Cloud were obtained. The details of the radial velocities derived from these plates are being prepared for publication elsewhere. To these velocities could be added the mean velocity of the great 30 Doradus nebula determined in an earlier investigation (Feast 1961) from Cassegrain observations at many different points 